\title{
A review on wearable photoplethysmography sensors and their potential future applications in health care
}

\begin{abstract}
Photoplethysmography (PPG) is an uncomplicated and inexpensive optical measurement method that is often used for heart rate monitoring purposes. PPG is a non-invasive technology that uses a light source and a photodetector at the surface of skin to measure the volumetric variations of blood circulation. Recently, there has been much interest from numerous researchers around the globe to extract further valuable information from the PPG signal in addition to heart rate estimation and pulse oxymetry readings. PPG signal's second derivative wave contains important health-related information. Thus, analysis of this waveform can help researchers and clinicians to evaluate various cardiovascular-related diseases such as atherosclerosis and arterial stiffness. Moreover, investigating the second derivative wave of PPG signal can also assist in early detection and diagnosis of various cardiovascular illnesses that may possibly appear later in life. For early recognition and analysis of such illnesses, continuous and real-time monitoring is an important approach that has been enabled by the latest technological advances in sensor technology and wireless communications. The aim of this article is to briefly consider some of the current developments and challenges of wearable PPG-based monitoring technologies and then to discuss some of the potential applications of this technology in clinical settings.
\end{abstract}

Volume 4 Issue 4 - 2018

\author{
Denisse Castaneda,' Aibhlin Esparza,' \\ Mohammad Ghamari, ${ }^{2}$ Cinna Soltanpur, ${ }^{3}$ \\ Homer Nazeran' \\ 'Department of Electrical and Computer Engineering, University \\ of Texas at El Paso, USA \\ ${ }^{2}$ Department of Energy and Mineral Engineering, Pennsylvania \\ State University, USA \\ ${ }^{3}$ Department of Electrical and Computer Engineering, University \\ of Oklahoma, USA
}

\begin{abstract}
Correspondence: Mohammad Ghamari, Department of Energy and Mineral Engineering, Pennsylvania State University, State College, 123 Student Health Center, University Park, PA 16802, Pennsylvania, USA, Tel: 9153834996,

Email: mug990@psu.edu
\end{abstract}

Received: July 17, 2018 | Published: August 06, 2018

\section{Introduction}

Wearable health monitoring technologies, including smart watches and fitness trackers, have attracted considerable consumer interest over the past few years. ${ }^{1-3}$ Not only has this interest has been mainly encouraged by the rapid demand growth in the wearable technology market for the ubiquitous, continuous, and pervasive monitoring of vital signs, but it has been leveraged by the state-of-the-art technological developments in sensor technology and wireless communications. ${ }^{4-7}$ According to Page, ${ }^{8}$ the wearable technology market was valued at over $\$ 13.2$ billion by the end of 2016 and its value is forecast to reach $\$ 34$ billion by the end of 2020 . Among the different categories on the wearable technology market, pervasive health monitoring applications are ranked the fastest growing segments due to the overwhelming need to monitor chronic diseases and aging populations ${ }^{9,10}$ Currently, modern wearable devices are no longer only focused on simple fitness tracking measurements such as the number of steps taken in a day, they also monitor important physiological considerations, such as Heart Rate Variability (HRV), glucose measures, blood pressure readings, and much additional health-related information. ${ }^{9}$ Among the numerous vital signs measured, the heart rate (HR) calculation has been one of the most valuable parameters. For many years, the Electrocardiogram (ECG) has been used as a dominant cardiac monitoring technique to identify cardiovascular abnormalities and to detect irregularities in heart rhythms. ${ }^{11}$ The ECG is a recording of the electrical activity of the heart. It shows the variations in the amplitude of the ECG signal versus time. This recorded electrical activity originates from the depolarization of conductive pathway of the heart and the cardiac muscle tissues during each cardiac cycle. ${ }^{12}$ Even though traditional cardiac monitoring technologies using the ECG signals has undergone continuous improvements for decades to address the ever-changing requirements of their users, specifically in terms of measurement accuracy and wearing comfort ability as shown in, ${ }^{13-16}$ these techniques, up to now, have not been enhanced to the point of offering the user flexibility, portability, and convenience. For instance, for the ECG to operate effectively, several bioelectrodes must be placed at certain body locations; this procedure greatly limits the moving flexibility and mobility of the users. In addition, PPG has shown itself to be an alternative HR monitoring technique. For instance, Bolaños et al., ${ }^{17}$ compared the HRV signals extracted from PPG and ECG signals. By using detailed signal analysis, they demonstrated that the PPG signal offers an excellent potential to replace ECG recordings for the extraction of HRV signals, especially in monitoring healthy individuals. Therefore, to overcome the ECG limitations, an alternative solution based on PPG technology can be used.

Photoplethysmography, known most commonly as PPG, utilizes an infrared light to measure the volumetric variations of blood circulation. This measurement provides valuable information about the cardiovascular system. ${ }^{18}$ The popularity of the PPG technology as an alternative heart rate monitoring technique has recently increased, mainly due to the simplicity of its operation, the wearing comfort ability for its users, and its cost effectiveness. ${ }^{19}$ However, one of the major difficulties in using PPG-based monitoring techniques is their inaccuracy in tracking the PPG signals during daily routine activities and light physical exercises. This limitation is due to the fact that the PPG signals are very susceptible to Motion Artifacts (MA) caused by hand movements. ${ }^{20}$ Moreover, alternative factors such as environmental noise may also affect the PPG signal acquisition, which consequently affect the estimation accuracy of the HR. ${ }^{21}$ Many studies have demonstrated that the second derivative of the PPG signal contains valuable health-related information. ${ }^{22}$ Investigation into this signal has shown strong potential to assist researchers and clinicians in evaluating various cardiovascular-related diseases, including atherosclerosis and arterial stiffness. In addition, the detailed 
analysis of this signal can also help with the timely identification and diagnose of various cardiovascular diseases. The goal of this review article is to investigate some valuable aspects of the PPG signal and PPG-based monitoring devices. The PPG's ability to measure blood variations in different parts of the body and its potential ability to detect physiological parameters that are linked to the cardiovascular and respiratory systems has continued to motivate the scientific community to develop more inexpensive and highly accurate wearable PPG-based devices for monitoring daily routine activities. Future research will continue to refine different techniques and approaches to reduce the effects of MA on the quality of the PPG signal.

\section{PPG-based monitoring devices}

A typical PPG device contains a light source and a photodetector. The light source emits light to a tissue and the photodetector measures the reflected light from the tissue. The reflected light is proportional to blood volume variations. ${ }^{23}$ Similar to ECGs, PPG waves can also help to diagnose cardiac arrhythmias (irregular heartbeat) because they reliably manifest cardiac and respiratory activities. Most common PPG sensors use an infrared light emitting diode (IRLED) or a green LED as the main light source. IR-LEDs are most commonly used for measuring the flow of blood that is more deeply concentrated in certain parts of body such as the muscles, whereas green light is typically used for calculating the absorption of oxygen in oxyhemoglobin (oxygenated blood) and deoxyhemoglobin (blood without oxygen present). ${ }^{24}$ Although there are other LED sensors with different colors to measure hemoglobin, green LED is considered the most commonly used. This is simply because it penetrates more deeply into tissue and therefore can provide measurements that are more accurate. PPG sensors also use a photodetector to measure the intensity of reflected light from the tissue. The blood volume changes can then be measured (calculated) based on the amount of the detected light. In addition, according to ${ }^{25} \mathrm{PPG}$ sensors are also useful in the determination of hyperemia, or an excess of blood flow. Wearable PPG sensors can only be placed at certain body locations as shown in Figure 1. However, different measurement sites have different degrees of accuracy. ${ }^{18}$ While it is most common to use specific body locations such as the finger, earlobe and forehead, researchers are considering other body locations for more convenient alternatives.

HR monitoring techniques that rely on PPG sensors have several advantages over traditional ECG-based systems. For instance, PPG sensors use simpler hardware implementation and have lower costs, and for operation, only a single sensor is required to be placed on the body. This is in contrast with traditional ECG recordings. ${ }^{26} \mathrm{~A}$ traditional ECG-based system requires at least three bioelectrodes placed on different body locations (such as the right arm, left arm and right leg) to be able to operate effectively. This requirement greatly restricts the patients' flexibility of motion. In addition, PPG sensors can operate more effectively if they are placed at specific easily accessible anatomical positions such as the earlobe and fingertip where the desired PPG signals are collected with higher quality. Consequently, it is imperative to find specific measurement sites that guarantee the optimal quality of sensor data. PPG sensors are designed in two different distinct forms: transmission mode and reflectance mode. Each mode comes with advantages and disadvantages. In transmission mode, the light source and detector are separated by the tissue, whereas in reflectance mode, the photodetector is positioned along the light source on the same side of the tissue to measure the reflected light. Both sensor types can provide non-invasive measurements. ${ }^{27}$ However, in transmission mode, too much pressure can slow down the peripheral blood volume, which may result in the reduction of venous oscillations. A measurement site is chosen based on different applications. ${ }^{28-30}$ For transmission mode, the fingertip and earlobe are commonly used. The measurement body placements for the reflectance mode sensors are the wrists, forearm, ankle, forehead and torso, as shown in Fig. 1. At different measurement sites, the sensors can either be used as cuffs or clips. The required amount of pressure to apply the sensor is also a key factor in selecting a specific measurement site. ${ }^{31}$

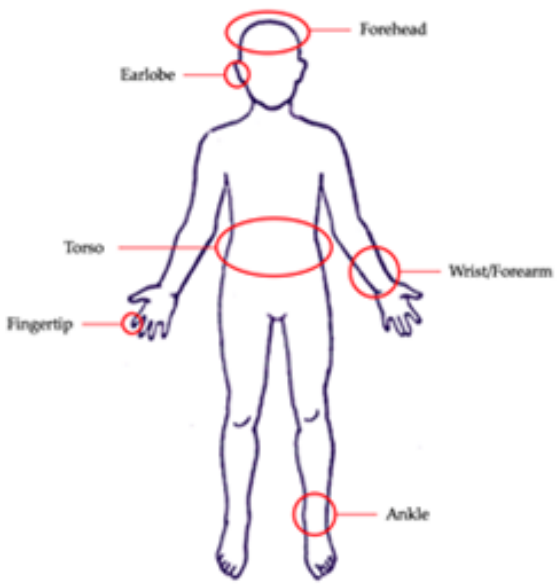

Figure IMost common measurement sites for PPG.

\section{Wristband-type PPG-based Devices}

In comparison to the various types of PPG-based HR monitoring devices in existence, the wristband-type PPG is considered the most popular and preferred device. The reason for its popularity is partly due to its remarkable properties such as being inexpensive, highly portable, and very convenient to wear by its users. However, these devices also have their own limitations. Several suggestions for tackling the shortcomings of wrist-type PPG devices for clinical setups have been presented in various studies to date. For instance, in Lee et al., ${ }^{32}$ presented a novel wristwatch PPG probe positioned on the ulnar and radial arteries in the patient's wrist instead of the blood capillaries as the common measurement site. The proposed device improved sensitivity and accuracy of the PPG signal by using an array of sensors, IR-LEDs, and photo transistors. Thomas et al., ${ }^{33}$ proposed a method to mitigate the effects of motion artifacts on the quality of the PPG signal. In this method, a nine-axis MEMS inertial sensor along with green LEDs were added to the PPG device to sense body measurements and detect posture. ${ }^{33} \mathrm{~A}$ similar method was also proposed $\mathrm{in}^{34}$ to mitigate motion artifacts by applying two reflective pulse signals from a single green LED sensor.

\section{Forehead-type PPG-based devices}

The human forehead can also be utilized as an alternative site for heart rate monitoring using a PPG device. Generally, the reflectance of the optical signal from a person's forehead is relatively powerful. This is because of the fact that the human skull is covered by comparatively thin skin along with a higher density of blood vessels in the forehead region. The placement of the reflectance mode PPG sensors on someone's forehead has shown an improved reaction to pulsatile signal variations in low perfusion environments. ${ }^{18}$ Previous studies such as ${ }^{18}$ have shown that the placement of PPG sensors on the human 
forehead can alleviate the destructive effects of motion artifacts on the quality of the PPG signal specially during light physical activities. Mendelson et al., ${ }^{35}$ used six photo detectors that were mounted on a soldier's helmet. They found that using minimal pressure between the sensors and tissue could produce less noisy signals from the person's forehead..$^{35}$

\section{Ear-type PPG-based devices}

The earlobe is one of the most frequently used measurement sites for PPG-based devices. This is due to the scientific fact that earlobes are not comprised of cartilage and thus they contain large blood supplies. Moreover, earlobes are far less vulnerable to the effects of motion artifacts compared to other extremities. Magnetic ear clips and headphones have been used in the past to obtain PPG signals. Poh et al., ${ }^{36}$ proposed a magnetic earring sensor to be placed on the earlobe. In addition to earrings, ear-type PPG sensors can also be designed and incorporated into an earphone and earbud to provide more comfort for the users. ${ }^{18}$ After a PPG sensor is placed in the ear, the sensor earbuds could be positioned against the tragus to be able to sense the light reflected from the subcutaneous blood vessels. ${ }^{18}$ Alternatively, a PPG sensor can be placed in the ear canal. Budidha et al., ${ }^{37}$ demonstrated that by placing a PPG sensor in the ear canal, a more accurate signal could be collected.

\section{PPG sensors}

Photoplethysmography sensors are designed in different types but they all measure changes in blood volume and provide similar results despite these differences in design. ${ }^{38} \mathrm{~A}$ typical PPG sensor emits light at the tissue site with one or more LEDs. The photodiode measures the intensity of the non-absorbed light reflected from the tissue. ${ }^{39}$ The LED colors used in most scientific trials are red and green; however, in some studies a yellow LED has also been used..$^{40}$ Light with longer wavelengths penetrates more deeply into the tissue. ${ }^{41}$ For instance, infrared light has a more effective penetration depth in the skin compared to green light. However, the authors in ${ }^{41}$ stated that infrared light is more susceptible to motion artifacts. Therefore, green LED that has shorter wavelength may be a better option for certain applications. ${ }^{41}$ Motion artifacts are usually caused by the movement of the PPG sensor over the tissue, skin deformation, blood flow dynamics, and ambient temperature. ${ }^{42,43}$ In addition, wearable devices could be equipped with accelerometers to capture the direction of motion to reduce movement artifacts, ${ }^{40}$ especially during intense physical activity.

\section{Factors affecting PPG sensor recordings}

Several factors can affect PPG recordings. These factors are sensing, biological, and cardiovascular factors. Table 1 gives a brief list of these factors. Tissue modifications generated by voluntary or involuntary movements can create alterations of inner tissues, such as muscle movement and dilation of tissues. The receiving light will be modified due to these movements, generating a different signal. The anatomy of individuals along with differences in organ sizes and amount of fluids retained by the tissues result in variation of the propagated light through the tissue. ${ }^{44}$ Another factor that can modify the signal is the displacement of the sensor. Physical activities and body movements may result in the displacement of the sensor relative to its original location. The sensor movement changes the path of light and consequently modifies the signals. ${ }^{45}$ The pressure applied by the device on the skin controls the magnitude of the received signal.
Table I Factors altering PPG response. ${ }^{45}$

\begin{tabular}{ll}
\hline & Sensor geometry \\
Sensing & Emitted light intensity \\
& Sensor-skin interface \\
& Ambient light \\
& Photodiode sensitivity \\
Biological & Oxygen concentration \\
& Organ characteristics \\
& Microcirculation volume \\
Cardiovascular & Arterial blood volume \\
& Interstitial fluids \\
& Venous volume \\
\hline
\end{tabular}

\section{PPG signal}

The PPG signal comprises pulsatile (AC) and superimposed (DC) components. The AC component is provided by the cardiac synchronous variations in blood volume that arise from heartbeats. The DC component is shaped by respiration, sympathetic nervous system activity, and thermoregulation. ${ }^{46}$ The $\mathrm{AC}$ component depicts changes in blood volume, which are caused by cardiac activity and depend on the systolic and diastolic phases..$^{47-49}$ The systolic phase (also called, "rise time") starts with a valley and ends with the pulse wave systolic peak. The pulse wave end is marked by another valley at the end of the diastolic phase..$^{51}$ Features such as rise time, amplitude, and shape can predict vascular changes in the bloodstream. ${ }^{52,53}$ Additionally, PPG can be used to measure HRV, ${ }^{54,55}$ or the variations between heartbeat time intervals (Peak-to-Peak or P-P Interval) as shown in Figure 2. The variation can be due to many factors such as the individual's age, heart conditions, and physical fitness. ${ }^{56} \mathrm{HRV}$ is used for evaluating the sympathetic and parasympathetic influences of the Autonomic Nervous System (ANS). ${ }^{57}$ Factors affecting HRV include, but are not limited to, age, cancer and thermoregulation [58], [59]. The PPG signal is divided into two unique phases: the rising edge of the pulse called anacrotic, which primarily describes the systole, and the falling edge of the pulse called the catacrotic, which represents the diastole. Additionally, a dicrotic notch, is typically visible at the catacrotic phase. $^{22}$ To ease the interpretation of the PPG wave, Ozawa et al differentiated the PPG signals to analyze the wave contour. ${ }^{60}$ Table 2 describes the main features of the original PPG signal.

Table 2 features of PPG signal22,50,61-64

\begin{tabular}{ll}
\hline PPG Feature & Description \\
\hline Systolic Amplitude & $\begin{array}{l}\text { Reflects AC variation in blood } \\
\text { volume around the measurement } \\
\text { site. } \\
\text { Total area under the PPG curve. } \\
\text { Interval between two systolic } \\
\text { peaks. }\end{array}$ \\
Peak to Peak Interval & $\begin{array}{l}\text { The time interval between the } \\
\text { systolic and diastolic peaks. }\end{array}$ \\
Large Artery Stiffness Index &
\end{tabular}

Figure 2 Sample of a photoplethysmogram signal where P-P interval is marked

\section{Second derivative wave of PPG signal}

The second derivative wave of the original PPG signal is 
called the acceleration photoplethysmogram (APG), and it is more commonly used than the first derivative wave. APG is an indicator of the acceleration of the blood. Figure 3 shows the original PPG signal along with its first and second derivative waves. ${ }^{57}$ There are a number of critical points that can be extracted from the second derivative wave of a PPG signal. These critical points can be used to detect and diagnose cardiac abnormalities. In clinical and research settings, there are still ongoing efforts to improve the current methods of obtaining critical points from the second derivative wave of the PPG signal. ${ }^{5,7,10}$ Figure 3 shows only three critical points that were extracted by ${ }^{57}$ from the original PPG signal. Other articles such as ${ }^{22}$ investigated additional critical points of the second derivative wave. As demonstrated in, ${ }^{22}$ critical point $a$ is the early systolic location. Point $b$ is the lowest point in the early systolic wave. Point $c$ is the resurgent of late systolic. Point $d$ indicates the decreasing part of late systolic and point $e$ represents the early diastolic wave. The APG main features for waveform analysis are described in more detail in Table 3. From the second derivative, we can compute the large artery stiffness index..$^{65}$ Additionally, the APG correlates with the distensibility of the carotid artery, age, blood pressure, risk of coronary heart disease, and the presence of the atherosclerotic disorders. $22,60,66-69$ PPG describes how fast blood moves within blood vessels. Systolic and diastolic waves interact with each other to form a waveform that resembles a long curve with varying troughs and rests that represent the critical points as stated before. The positive waves, namely the a, c, and e waves, rest above the baseline and have positive values, while $b$ and $d$ are negative waves. Thus, the latter waves lie below the baseline due to their negative values. The relationship between the waves represents different physiological trends found in subjects. For example, the ratio b/a represents increased arterial stiffness that increases with age. ${ }^{22}$ This ratio can also indicate hypertension. Potential work includes examining the relationship between $\mathrm{a} / \mathrm{b}$ and studying the impact of age, body mass index, and core temperature on PPG waves. ${ }^{75}$ To date, there are algorithms that can detect a-waves and b-waves, but not accurately. In order to analyze the results of a PPG experiment, there needs to be a clear and accurate assessment of these waves to determine future steps to be taken for the assessment of arterial stiffness and other cardiovascular diseases that may be present. ${ }^{75}$

Table 3 acceleration photoplethysmogram features ${ }^{22,60,70-74}$

\begin{tabular}{ll}
\hline APG Features & Description \\
\hline Ratio c/a, e/a & Indicates arterial stiffness. \\
Ratio b/a & $\begin{array}{l}\text { Reflects increased arterial stiffness, } \\
\text { consequently increases with age. } \\
\text { Indicates decreased arterial stiffness. } \\
\text { Useful parameter for the evaluation of left } \\
\text { ventricular afterload. } \\
\text { Ratio d/a }\end{array}$ \\
Ratio (b-c-d-e)/a & $\begin{array}{l}\text { arteriosclerotic disease indicator. } \\
\text { Ratio (b-e)/a }\end{array}$ \\
Ratio (c+d-b)/a & $\begin{array}{l}\text { A more comprehensive aging index. } \\
\text { Represents a completed cardiac cycle. HRV } \\
\text { can be calculated using the a-a interval. }\end{array}$ \\
\hline
\end{tabular}

\section{Some PPG applications}

The early detection of physiological parameters based on PPG signals has become of great interest to the research and clinical community. Because PPG is an indication of the blood flow generated by the heart using near-infrared light, this method can be used to detect cardiovascular diseases, such as vascular aging. The cardiovascular and respiratory systems work together and due to this synergistic relationship; PPG offers the possibility of obtaining respiratory related information. The section below goes into detail on how PPG could potentially collect information related to vascular aging and respiratory physiology.

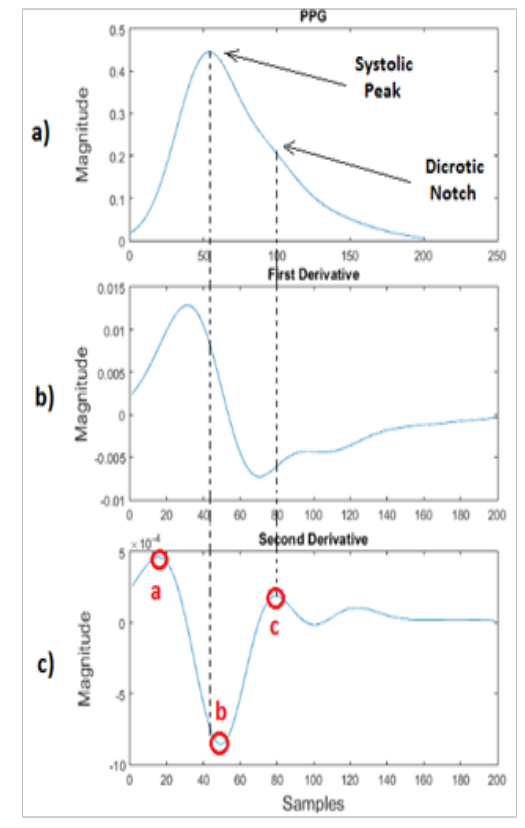

Figure 3 A) PPG signal B) PPG first derivative C) PPG second derivative.

\section{Vascular aging and PPG}

Ageing is one of the factors that can lead to arterial stiffness because of the noticeable changes in peripheral pulse propagations. In younger subjects, such propagations reveal a steep systolic peak. ${ }^{76}$ This means that the presence of ageing is barely visible in young subjects, but compared to older subjects, the systolic peak will be visibly steeper. Arterial stiffness is a flag for cardiovascular diseases which will show up on the pulse timing in the PPG signal. Peripheral pulse can predict whether or not arterial stiffness is present and can also predict future cardiovascular problems because it is a biomarker for the assessment of health and disease..$^{77}$ As an individual gets older, the arteries get larger and less dense: this change is reflected where the wave peaks in the PPG signal. ${ }^{77}$ By evaluating different points and magnitudes of the PPG signal, which reflects arterial wall stiffness, the pumping power of the left ventricle can be analyzed..$^{77}$ The amplitude of the PPG can show changes in blood volume, thereby giving information about arterial compliance and arterial elastic properties. ${ }^{77}$ With increased arterial stiffness, the vessel thickness increases and the inner diameter is reduced, which makes it harder for the patient's cardiovascular system to work. The volume of blood moved in a given time provides an indication of vascular aging during the cardiac cycle. ${ }^{76}$ The maximum amplitude of a single pulse denotes the relationship between age and arterial stiffness. Arteriosclerosis thickens and hardens the walls of arteries. Consequently, their resistance becomes higher and their capacitance declines. ${ }^{77}$ Another important feature in analyzing PPG signals is to assess how well blood vessels adapt to 
their environment and more specifically, to the thickness of the blood in the cardiac system. ${ }^{76}$ Age plays a crucial role in arterial stiffness as arteriosclerosis occurs with older adulthood. ${ }^{77}$ However, it is still difficult to get a clear detection of the waves, due to blurred inflection points, making it hard to determine where arterial stiffness is located in the PPG signal. ${ }^{78}$ The second derivative is used to monitor arterial conditions such as the vascular response in resistance arteries, which are important in regulating blood pressure. ${ }^{79,80}$ The stiffness index is computed by taking the body height and dividing it by the interval between the systolic and diastolic peaks. ${ }^{22,48}$ Vascular aging can be evaluated through the SDPPG aging index, SDPPG-AI (b-c-d-e/a). ${ }^{81}$ The above-mentioned index shows that aging causes arterial dilation and stiffness By setting a relationship between $a$ and $b$ parameters, valuable information can be extracted. For instance, it has been shown in $^{22}$ that $\mathrm{b} / \mathrm{a}$ relationship increases with age and the $\mathrm{d} / \mathrm{a}$ relationship decreases with age.

\section{Respiration rate and PPG}

Vital signs, of which respiratory rate is one of the essential components, are critical in determining a subject's health and potential illnesses. ${ }^{96}$ Respiratory rate is the number of breaths a person takes per minute while resting. ${ }^{44}$ Respiratory rates can be at a healthy level, or too high or too low. ${ }^{44}$ Current devices used to determine respiratory rates include a nasal cannula and a chest band, but these methods can be harmful to the patient. ${ }^{44}$ Respiratory rates are related to PPG in three ways: 1) The pulse wave amplitude is affected by the flexibility of the blood vessels, 2) there is a variation of the pulse envelope, and 3 ) a decrease in intrathoracic pressure can lead to increasing venous return during inspiration. ${ }^{44}$ Using PPG to estimate respiration rates could be a potential approach for obtaining information on respirationrelated matters. PPG could be used to extract or identify a respiratory trend embedded in physiological signals..$^{82}$ There are three respiratoryinduced variations that can be extracted from a PPG; frequency, intensity, and amplitude..$^{83}$ The frequency and amplitude of the heartrelated variations are modulated by respiration which changes the statistical characteristics of the signal. The modulated signal has a non-stationary nature, which in turn causes difficulty in the estimation of HRV ${ }^{84,85}$ A method proposed by Chon et al. ${ }^{86}$ refers to a technique that utilizes the pulse oximeter signal to estimate respiratory rate. ${ }^{86}$ The proposed variable-frequency complex demodulation (VFCDM) provides accurate time, greater resolution, and better amplitude estimates compared to other methods, such as the continuous wavelet transform (CWT), and autoregressive (AR) modeling. ${ }^{86}$

\section{Discussion}

Monitoring of heart rate during daily routine activities and physical exercise is an important feature in many modern wearable devices such as wristbands and smart watches. However, obtaining high quality PPG signals during physical exercise is difficult and challenging as PPG signals are usually contaminated by very strong motion artifacts caused by subject's hand movements. This area of research has been very popular for the past few years and many leading high-tech companies and academics have been actively working on this topic. Currently researchers are investigating the effects of motion artifacts on the quality of acquired PPG signals and proposing solutions to mitigate or ideally remove this destructive affects. Examples of highly cited articles that use signal-processing approaches to tackle this problem are shown in..$^{87,88,89,90,91} \mathrm{~A}$ vast number of articles in this topic as shown in, ${ }^{93,93,94,95}$ also use accelerometer data in order to be able to remove the motion artifact problem. In addition, many researchers such as, ${ }^{22,96,97}$ nowadays around the globe are investigating to possibly extract further valuable information from the PPG signal in addition to heart rate estimation and pulse oxymetry readings. This paper in particular considered articles that investigates the second derivative wave of original PPG signal. We investigated how second derivative wave can be used to estimate the vascular aging and compared attempts that have been done in the past by other researchers to monitor arterial conditions such as. ${ }^{60,69,79,80}$

\section{Conclusion}

PPG is a noninvasive, low cost, and simple optical measurement technique applied at the surface of the skin to measure physiological parameters. Scientific interest has continued to look beyond the pulse oximetry and heart rate calculation, and more into the potential applications of PPG sensors. It is now well known that the second derivative wave of the original PPG signal contains important health-related information and the analysis of this wave could lead researchers, clinicians, and health-care providers to the early detection and diagnosis of various cardiovascular diseases typically occurring later in life. In processing the acceleration of the PPG signal, troughs and rests carry valuable health-related information that can be used by health-care professionals to learn about the well-being of the patient's heart and cardiovascular system. Through filtering and feature extraction, a specific wave can be targeted, and its patterns correlating to physiological biomarkers can be determined. PPG thus reveals itself as a promising technology in both health-care settings and in assessment of daily activity, due to its non-invasiveness, low cost, and portability. It has the potential to furnish health-care providers with the tool that will allow the early detection and diagnosis of cardiovascular diseases, thereby offering greater insight into a patient's health. However, further investigations using low power consumption to determine even more vital health-related information must be conducted.

\section{Acknowledgements}

This work was supported by the National Institute of Heath Diversity Program Consortium through BUILD award numbers 8UL1GM118970-02 and 8RL5GM118969-02.

\section{Conflict of interest}

Authors declares that there is no conflict of interest.

\section{References}

1. Rawassizadeh R, Price BA, Petre M. Wearables: has the age of smartwatches finally arrived? Commun ACM. 2015;58(1):45-47.

2. Wei J. How wearables intersect with the cloud and the internet of things: Considerations for the developers of wearables. IEEE Consum Electron Mag. 2014;3(3):53-56.

3. Islam SMR, Kwak D, Kabir H, et al. The Internet of Things for Health Care : A Comprehensive Survey. IEEE. 2015;3:678-708.

4. Ranck J. The wearable computing market : a global analysis. Gigaom Pro. 2012;1-26.

5. Chan M, Estève D, Fourniols JY, et al. Smart wearable systems: Current status and future challenges. Artif Intell Med. 2012;56(3):137-156.

6. Ghamari M, Janko B, Sherratt RS, et al. A survey on wireless body area networks for ehealthcare systems in residential environments. Sensors. $2016 ; 16(6): 1-33$. 
7. Ghamari M, Arora H, Sherratt RS, et al. Comparison of low-power wireless communication technologies for wearable health-monitoring applications. I4CT 2015 - 2015 2nd International Conference on Computer, Communications, and Control Technology, Art Proceeding. 2015:1-6.

8. Page T. A Forecast of the Adoption of Wearable Technology. Int J Technol Diffus. 2015;6(2):12-29.

9. Park S, Jayaraman S. Enhancing the Quality of Life Through Wearable Technology. IEEE Eng Med Biol Mag. 2003;22(3):41-48.

10. Pantelopoulos A, Bourbakis NG. survey on wearable sensor-based system for health monitoring and prognosis. IEEE Trans Syst Man Cybern Part C Appl Rev. 2010;40(1):1-12.

11. Al Ghatrif M, Lindsay J. A brief review: history to understand fundamentals of electrocardiography. $J$ Community Hosp Intern Med Perspect. 2012;2(1):14383.

12. Bolanos M, Nazeran H, Haltiwanger E. Comparison of heart rate variability signal features derived from electrocardiography and photoplethysmography in healthy individuals. International Conference of the IEEE Engineering in Medicine and Biology Society. 2006;1:42894294.

13. Mc Laughlin NB, Campbell RW, Murray A. Accuracy of four automatic QT measurement techniques in cardiac patients and healthy subjects. Heart. 1996;76(5):422-6.

14. Postema PG, De Jong JS, Van der Bilt IA, et al. Accurate electrocardiographic assessment of the QT interval: Teach the tangent Hear Rhythm, 2008;5(7):1015-1018.

15. Nemati E, Jamal Deem M, Mondal T. A wireless wearable ECG sensor for long-term applications. IEEE Commun Mag. 2012;50(1):36-43.

16. Park C, Chou PH, Bai Y, et al. An ultra-wearable, wireless, low power ECG monitoring system. 2006:241-244.

17. Bolanos M, Nazeran H, Haltiwanger E, et al. Comparison of heart rate variability signal features derived from electrocardiography and photoplethysmography in healthy individuals. Engineering in Medicine and Biology Society. 2006;1:4289-4294.

18. Tamura T, Maeda Y, Sekine M, et al. Wearable photoplethysmographic sensors - past and present. Electronics. 2014;3:282-302.

19. Sviridova N, Sakai K. Human photoplethysmogram: new insight into chaotic characteristics. Chaos Solitons \& Fractals. 2015;77:53-63.

20.Zhang Y, Liu B, Zhang. Combining ensemble empirical mode decomposition with spectrum subtraction technique for heart rate monitoring using wrist-type photoplethysmography. Biomed Signal Process Control. 2015;21:119-125.

21.Zhang Z, Pi Z, Liu B. TROIKA: a general framework for heart rate monitoring using wrist-type photoplethysmographic signals during intensive physical exercise. IEEE Trans Biomed Eng. 2015;62(2):522-531.

22. Elgendi M. On the analysis of fingertip photoplethysmogram signals. Curr Cardiol Rev. 2012;8(1): 14-25.

23. Wang C, Li Z, Wei X. Monitoring heart and respiratory rates at radial artery based on PPG. Opt Int J Light Electron Opt. 2013;124(4):3954-3956.

24. Kavsaoğlu AR, Polat K, Hariharan M. Non-invasive prediction of hemoglobin level using machine learning techniques with the PPG signal's characteristics features. Appl Soft Comput. 2015;37:983-991.

25. John D Enderle, Bronzino JD. Introduction to biomedical engineering. Elsevier/Academic Press, 2012.

26.Zhang Z. Photoplethysmography-based heart rate monitoring in physical activities via joint sparse spectrum reconstruction. IEEE Trans Biomed Eng. 2015;62(8):1902-1910.
27. Allen J, Murray A. Similarity in bilateral photoplethysmographic peripheral pulse wave characteristics at the ears, thumbs and toes. Physiol Meas. 2000;21(3):369-377.

28. Nilsson L, Johansson A, Kalman S, et al. Respiration can be monitored by Photoplethysmography with high sensitivity and specificity regardless of anaesthesia and ventilatory mode. Acta Anaesthesiol Scand. 2005;49(8):1157-1162

29. Jönsson B, Laurent C, Skau T, et al. A new probe for ankle systolic pressure measurement using Photoplethysmography (PPG). Ann Biomed Eng. $2005 ; 33(2): 232-9$

30. Shelley KH, Tamai D, Jablonka D, et al. The effect of venous pulsation on the forehead pulse oximeter wave form as a possible source of error in Spo2 calculation. Anesth Analg. 2005;100(3):743-747.

31. Renevey P, Sola J, Theurillat P, et al. Validation of a wrist monitor for accurate estimation of RR intervals during sleep. 35th Annual International Conference of the IEEE Engineering in Medicine and Biology Society (EMBC). 2013:5493-5496.

32. Lee Y, Shin H, Jo J, et al. Development of a wristwatch-type PPG array sensor module. IEEE International Conference on Consumer Electronics -Berlin (ICCE-Berlin). 2011:168-171.

33. Thomas SS, Nathan V, Zong C, et al. BioWatch: A noninvasive wrist-based blood pressure monitor that incorporates training techniques for posture and subject variability. IEEE J Biomed Health Inform. 2016;20(5):1291300 .

34. Lee H, Ko H, Jeong C, et al. Wearable photoplethysmographic sensor based on different LED light intensities. IEEE Sens J. 2016:1.

35. Mendelson Y, Pujary C. Measurement site and photodetector size considerations in optimizing power consumption of a wearable reflectance pulse oximeter. Proceedings of the 25th Annual International Conference of the IEEE Engineering in Medicine and Biology Society (IEEE Cat. No.03CH37439). 2003:3016-3019.

36. Poh MG, Swenson NC, Picard RW. Motion-tolerant magnetic earring sensor and wireless earpiece for wearable Photoplethysmography. IEEE Trans Inf Technol Biomed. 2010;14(3):786-794.

37. Budidha K, Kyriacou PA. The human ear canal: investigation of it suitability for monitoring photoplethysmographs and arterial oxygen saturation. Physiol Meas. 2014;35(2):111-128.

38. Cheang PR, Peck YS, Smith. An overview of non-contact photoplethysmography. Electron Syst Control Div Res. 2003.

39. Fischer C, Domer B, Wibmer T, et al. An Algorithm for Real-Time Pulse Waveform Segmentation and Artifact Detection in photoplethysmograms. IEEE J Biomed Heal Informatics. 2017;21(2):372-381.

40. Lai PH, Kim I. Lightweight wrist Photoplethysmography for heavy exercise: motion robust heart rate monitoring algorithm.

41. Spigulis J, Gailite L, Lihachev A, et al. Simultaneous recording of skin blood pulsations at different vascular depths by multiwavelength Photoplethysmography. Appl Opt. 2007;46(10):1754-1759.

42. Wijshoff RW, Mischi M, Veen J, et al. Reducing motion artifacts in photoplethysmograms by using relative sensor motion: phantom study. $J$ Biomed Opt. 2012;17(11):117007.

43. Tăuţan AM, Young A, Wentink E, et al. Characterization and reduction of motion artifacts in photoplethysmographic signals from a wrist-worn device. 37th Annual International Conference of the IEEE Engineering in Medicine and Biology Society (EMBC). 2015:6146-6149.

44. Sazonov E, Neuman MR. Wearable sensors: fundamentals, implementation and applications. San Diego: Academic Press, 2014

45. Lemay M, Bertschi M, Sola J, et al. Chapter 2.3. Application of Optical Heart Rate Monitoring. 2014. 
46. Allen J. Photoplethysmography and its application in clinical physiological measurement. Physiol Meas. 2007;28(3):R1-R39.

47. Allen J, Murray A. Effects of filtering on multi-site Photoplethysmography pulse waveform characteristics. Computers in Cardiology. 2004:485-488.

48. Gonzalez R, Manzo A, Delgado J, et al. A computer based photoplethysmographic vascular analyzer through derivatives. Computers in Cardiology. 2008:177-180.

49. Kyriazis J, Katsipi I, Stylianou K, et al. Arterial stiffness alterations during hemodialysis: the role of dialysate calcium. Nephron Clin Pract. 106(1):c34-c42.

50. Millasseau SC, Kelly RP, Ritter JM, et al. Determination of age-related increases in large artery stiffness by digital pulse contour analysis. Clin Sci (Lond). 2002;103(4):371-377.

51. Weissler AM, Harris WS, Schoenfeld CD. Systolic time intervals in heart failure in man. Circulation. 1968;37(2):149-159.

52. Oliva I, Roztoĉil K. Toe pulse wave analysis in obliterating atherosclerosis Angiology. 1983;34(9):610-619.

53. Allen J, Overbeck K, Nath AF, et al. A prospective comparison of bilateral photoplethysmography versus the ankle-brachial pressure index for detecting and quantifying lower limb peripheral arterial disease. $J$ Vasc Surg. 2008;47(4):794-802

54. Lu S, Zhao H, Ju K, et al. Can photoplethysmography variability serve as an alternative approach to obtain heart rate variability information? J Clin Monit Comput. 2008;22(1):23-29.

55. Nitzan M, Babchenko A, Khanokh B, et al. The variability of the photoplethysmographic signal--a potential method for the evaluation of the autonomic nervous system. Physiol Meas. 1998;19(1):93-102.

56. Lu G, Yang F, Taylor JA, et al. A comparison of photoplethysmography and ECG recording to analyse heart rate variability in healthy subjects. $J$ Med Eng Technol. 2009;33(8):634-41.

57. Ghamari M, Soltanpur C, Cabrera S, et al. Design and prototyping of a wristband - type wireless photoplethysmographic device for heart rate variability signal analysis. Conf Proc IEEE Eng Med Biol Soc. 2016;2016:4967-4970.

58. Stauss HM. Heart rate variability. American Journal of PhysiologyRegulatory, Integrative and Comparative Physiology. 2003;285(5):R927R931

59. Rompelman O, Coenen AJ, Kitney RI, et al. Measurement of heart-rate variability: part 1-comparative study of heart-rate variability analysis methods. Med Biol Eng Comput. 1977;15(3):233-239.

60. Takazawa K, Tanaka N, Fujita M, et al. Assessment of vasoactive agents and vascular aging by the second derivative of photoplethysmogram waveform. Hypertension. 1998;32(2):365-370.

61. Asada HH, Shaltis P, Reisner A, et al. Mobile monitoring with wearable photoplethysmographic biosensors. IEEE Eng Med Biol Mag. $2003 ; 22(3): 28-40$

62. Chua CP, Heneghan C. Continuous blood pressure monitoring using ECG and finger photoplethysmogram. International Conference of the IEEE Engineering in Medicine and Biology Society. 2006; 1:5117-5120.

63. Awad AA, Haddadin AS, Tantawy H, et al. The relationship between the photoplethysmographic waveform and systemic vascular resistance. J Clin Monit Comput. 2007;21(6):365-372.

64. Seitsonen ER, Korhonen IK, Van Gils MJ. EEG spectral entropy, heart rate, Photoplethysmography and motor responses to skin incision during sevoflurane anaesthesia. Acta Anaesthesiol Scand. 2005;49(3):284-292.
65. Ahn JM. New Aging Index Using Signal Features of Both Photoplethysmograms and Acceleration Plethysmograms. Healthc Inform Res. 2017;23(1):53-59.

66. Imanaga I, Hara H, Koyanagi S, et al. Correlation between wave components of the second derivative of Plethysmogram and arterial distensibility. Jpn Heart J. 1998;39(6):775-784

67. Hashimoto J, Chonan K, Aoki Y, et al. Pulse wave velocity and the second derivative of the finger photoplethysmogram in treated hypertensive patients: their relationship and associating factors. 2002;20(12):24152422.

68. Otsuka T, Kawada T, Katsumata M, et al. Utility of second derivative of the finger photoplethysmogram for the estimation of the risk of coronary heart disease in the general population. Circ J. 2006;70(3):304-310.

69. Bortolotto LA, Blacher J, Kondo T, et al. Assessment of vascular aging and atherosclerosis in hypertensive subjects: second derivative of photoplethysmogram versus pulse wave velocity. Am J Hypertens 2000;13(2):165-171.

70. Ushiroyama T, Kajimoto Y, Sakuma K, et al. Assessment of chilly sensation in Japanese women with laser doppler fluxmetry and Acceleration Plethysmogram with respect to peripheral circulation. 2005.

71. Elgendi M, Jonkman M, Deboer F. Heart rate variability measurement using the second derivative photoplethysmogram. 2010.

72. Elgendi MY, Jonkman ME, De Boer F. Measurement of a-a Intervals a Rest in the Second Derivative Plethysmogram. IEEE Int Symp Bioelectron. Bioinforma. 2009:76-79.

73. Elgendi MY, Jonkman ME, De Boer F. Applying the APG to measure heart rate variability. The 2nd International Conference on Computer and Automation Engineering (ICCAE), 2010:514-517.

74. Elgendi MY, Jonkman ME, De Boer F. Heart rate variability and the acceleration Plethysmogram signals measured at rest. International Joint Conference on Biomedical Engineering Systems and Technologies. 2011:266-277.

75. Elgendi M. Detection of $\mathrm{c}, \mathrm{d}$, and $\mathrm{e}$ waves in the acceleration photoplethysmogram. Comput Methods Programs Biomed. 2014;117(2):125-136.

76. Zahedi E, Chellappan K, Ali MA, et al. Analysis of the Effect of Ageing on Rising Edge Characteristics of the photoplethysmogram using a Modified Windkessel Mode. Cardiovasc Eng. 2007;7(4):172-181.

77. Yousef Q. The Analysis of PPG Morphology: Investigating the Effects of Aging on Arterial Compliance. Meas Sci Rev. 2012;12(6):271.

78. Wang L, Pickwell-Macpherson E, Liang YP, et al. Noninvasive cardiac output estimation using a novel photoplethysmogram index. Conf Proc IEEE Eng Med Biol Soc. 2009:1746-1749.

79. Baek HJ, Ki JS, Kim YS, et al. Second Derivative of Photoplethysmography for Estimating Vascular Aging. 6th International Special Topic Conference on Information Technology Applications in Biomedicine. 2007:70-72.

80. Mohamad Rozi R, Usman S, Mohd Ali MA, et al. Second derivatives of photoplethysmography (PPG) for estimating vascular aging of atherosclerotic patients. IEEE-EMBS Conference on Biomedical Engineering and Sciences. 2012:256-259.

81. Hyun JB, Jung SK, Yun SK, et al. Second derivative of Photoplethysmography for estimating vascular aging. Proc IEEE/EMBS Reg 8 Int Conf Inf Technol Appl Biomed. 2008:70-72.

82. Madhav KV, Ram MR, Krishna EH, et al. Estimation of respiration rate from ECG, BP and PPG signals using empirical mode decomposition. IEEE International Instrumentation and Measurement Technology Conference. 2011:1-4. 
83. Karlen W, Raman S, Ansermino JM, et al. Multiparameter Respiratory Rate Estimation From the photoplethysmogram. IEEE Trans Biomed Eng. 2013;60(7):1946-1953.

84. Nemati S, Malhotra A, Clifford GD. Data Fusion for Improved Respiration Rate Estimation. EURASIP J Adv Signal Process. 2010:926305.

85. Shokoueinejad M, Fernandez C, Carroll E, et al. Sleep apnea: a review of diagnostic sensors, algorithms, and therapies. Physiological Measurement. 2017;38(9):R204-R252.

86. Chon, KH, Dash S, Kihwan Ju. Estimation of Respiratory Rate From Photoplethysmogram Data Using Time-Frequency Spectral Estimation. IEEE Trans Biomed Eng. 2009;56(8):2054-2063.

87. Kim BS, Yoo Sk. Motion artifact reduction in photoplethysmography using independent component analysis. IEEE Transactions on Biomedical Engineering. 2006;53(3):566-568.

88. Lee CM, Zhang YT. Reduction of motion artifacts from photoplethysmographic recordings using a wavelet denoising approach. IEEE EMBS Asian-Pacific Conference on Biomedical Engineering. 2003:194-195.

89. Ram MR, MadhavKV, Krishna Eh, et al. A Novel Approach for Motion Artifact Reduction in PPG Signals Based on AS-LMS Adaptive Filter. IEEE Transactions on Instrumentation and Measurement. 2012;6(5):1445-1457.

90. Jianchu Yao, Warren S. A novel algorithm to separate motion artifacts from photoplethysmographic signals obtained with a reflectance pulse oximeter Conf Proc IEEE Eng Med Biol Soc. 2004;3:2153-2156.

91. Chan KW, Zhang Yt. Adaptive reduction of motion artifact from photoplethysmographic recordings using a variable step-size LMS filter. Sensors. 2002;2:1343-1346.
92. Asada HH, Hong-Hui Jiang, Gibbs P. Active noise cancellation using MEMS accelerometers for motion-tolerant wearable bio-sensors. The 26th Annual International Conference of the IEEE Engineering in Medicine and Biology Society, San Francisco. 2004:2157-2160.

93. Lee B, Han J, Baek HJ, et al. Improved elimination of motion artifacts from a photoplethysmographic signal using a Kalman smoother with simultaneous accelerometry. Physiological measurement. 2010;31(12):1585.

94. Kim SH, Ryoo DW, Bae C. Adaptive Noise Cancellation Using Accelerometers for the PPG Signal from Forehead. 2007 29th Annual International Conference of the IEEE Engineering in Medicine and Biology Society. 2007:2564-2567.

95. Fukushima H, Kawanaka H, Bhuiyan MS, et al. Estimating heart rate using wrist-type Photoplethysmography and acceleration sensor while running. Annual International Conference of the IEEE Engineering in Medicine and Biology Society. 2012:2901-2904.

96. Reșit Kavsaoğlu A, Polat K, Recep Bozkurt M. A novel feature ranking algorithm for biometric recognition with PPG signals. Comput Biol Med. 2014;49:1-14

97. Wei C, Sheng L, Lihua G, et al. Study on conditioning and feature extraction algorithm of photoplethysmography signal for physiological parameters detection. 2011 4th International Congress on Image and Signal Processing. 2011: 2194-2197. 